Service social

\title{
Des enjeux pour l'action militante des années 80
}

\section{André Jacob}

Volume 34, numéro 2-3, 1985

L'organisation communautaire

URI : https://id.erudit.org/iderudit/706278ar

DOI : https://doi.org/10.7202/706278ar

Aller au sommaire du numéro

Éditeur(s)

École de service social de l'Université Laval

ISSN

1708-1734 (numérique)

Découvrir la revue

Citer cet article

Jacob, A. (1985). Des enjeux pour l'action militante des années 80. Service social, 34(2-3), 353-368. https://doi.org/10.7202/706278ar d'utilisation que vous pouvez consulter en ligne.

https://apropos.erudit.org/fr/usagers/politique-dutilisation/ 
JACOB, André, professeur au Département de travail social de l'Université du Québec à Montréal.

\section{Des enjeux pour l'action militante des années 80}

\section{André Jacob}

Si les années 1960 ont été caractérisées par l'action militante de quartier et par les grands projets de participation populaire dans les régions rurales comme le Saguenay/Lac-Saint-Jean (TÉVEC) et l'Est du Québec (B.A.E.Q.), les années 1970 ont signifié une action militante beaucoup plus radicale, avec l'apparition d'une poussée gauchiste importante de tendance marxiste-léniniste, et la mise en place des nouvelles structures organisationnelles de l'État dans divers secteurs comme l'éducation et les services sociaux et de santé.

Évidemment, il apparaît un peu simpliste de caractériser ainsi deux décennies importantes pour l'histoire du mouvement populaire et de l'action militante d'après-guerre, au Québec. Notre objectif n'est pas de faire l'analyse des pratiques fort riches et mouvementées dans tous les secteurs reliés à l'organisation communautaire militante, mais plutôt d'identifier et d'analyser quelques enjeux actuels créés par la conjoncture sociale, politique, économique et idéologique des années 1980. Ces enjeux sont au cœur de la signification de l'action militante de la décennie que nous sommes en train de vivre et donnent une signification aux objectifs et aux stratégies de l'action militante, tant dans les organisations de masse que dans le mouvement ouvrier.

Dans un premier temps, il s'agit de clarifier la notion d'enjeu et sa signification dans la conjoncture actuelle. Deuxièmement, en partant de différentes luttes, l'analyse portera sur divers niveaux d'enjeux selon qu'ils se rattachent aux droits fondamentaux ayant une portée nationale, aux droits socio-économiques ou aux droits socio-politiques. 


\section{La signification des enjeux}

II ne s'agit pas ici de réinventer la roue en définissant la notion d'enjeu, puisque d'autres l'ont fait bien avant nous comme le Groupe de recherche en action populaire (Doré, 1981, pp. 21-23). Par ailleurs, il est justifié de situer le concept par rapport à la dynamique de l'action communautaire militante du milieu des années 1980.

Si, un enjeu, c'est l'objet d'une lutte au cœur d'intérêts opposés, on comprend aisément qu'une analyse de ceux-ci permette de situer les intérêts en cause dans une situation sociale qui commande une action, d'en voir les implications et de mener une lutte consciente et significative. En fait, une action doit s'appuyer sur une compréhension claire des enjeux au cœur de la lutte si elle prétend défendre les intérêts de la classe ouvrière et de la masse populaire. Évidemment, une telle analyse doit chercher à comprendre les intérêts de celles-ci ainsi que ceux de la classe dominante.

\section{Au coeur d'intérêts opposés}

La première question à se poser est : est-ce que l'ensemble de ses aspirations spontanées d'une classe sociale constitue nécessairement ses véritables intérêts? Par exemple, une grève ou une manifestation utilisées strictement comme moyens de pression pour exprimer des aspirations revendicatives, sans qu'on cherche à analyser les causes de la situation sociale qui fait l'objet de l'action, sans identifier les in térêts en cause (ceux de la classe dominante et ceux de la classe ouvrière et de la masse populaire) et sans remettre en question le système capitaliste peuvent-elles être des stratégies qui expriment vraiment les intérêts des couches sociales dominées?

Dans un processus d'action, comme celui mentionné dans l'exemple précédent, il faut surtout parler des "intérêts spontanés immédiats" (Harnecker, 1974, p. 161) ou des aspirations que manifestent les classes sociales face à des problèmes existentiels et actuels. En général, l'objectif visé sera d'obtenir un plus grand bien-être immédiat ou une meilleure répartition des richesses sociales. D'ailleurs, il ne s'agit pas là d'une simple considération théorique; il est tout à fait réaliste de considérer que l'intérêt immédiat d'un groupe d'ouvriers à bas revenus est d'obtenir une augmentation de salaire afin de pouvoir faire face rapidement à la hausse constante du coût de la vie. Il est tout aussi normal et spontané de voir des locataires protester contre la destruction du quartier auquel ils s'identifient. 
Par ailleurs, il importe de réaliser que les intérêts immédiats masquent souvent les enjeux fondamentaux d'une lutte parce qu'il sont influencés par l'idéologie dominante et ne s'en tiennent qu'à des stratégies réformistes. Par exemple, dans le mouvement ouvrier des pays capitalistes avancés, les luttes revendicatives sur les salaires sont souvent les seules valorisées, laissant de côté "l'élément conscient".

Souvent les revendications salariales ne sont pas liées à d'autres demandes, ni à d'autres secteurs de la vie : l'intellectuel, le social, le politique ou l'idéologique. Pour bien en comprendre la signification, ne faut-il pas les situer dans un rapport dialectique autour d'enjeux ou d'intérêts collectifs et globaux opposés?

De ce qui précède, on peut conclure que seuls les intérêts stratégiques à long terme représentent vraiment ceux des classes dominées; par contre, on ne peut bien identifier les enjeux et leurs vrais intérêts de classe qu'en partant des intérêts immédiats. On ne peut absolument pas masquer les intérêts particuliers, conjoncturels par des revendications purement politiques, globales, compréhensibles par les seuls(es) intellectuels(les), par les seuls(es) professionnels(les) de l'action politique ou par "l'avant-garde éclairée".

\section{Des enjeux plus économico-politiques}

Dans la conjoncture actuelle, les luttes sont de plus en plus politiques en raison de l'extension de la domination du capital monopoliste dans tous les secteurs (autant financier qu'industriel), y compris sur la scène politique. Les entreprises monopolistes exercent des pressions de plus en plus fortes sur l'ensemble des couches populaires par une inflation chronique, une augmentation croissante des impôts et des taxes de vente, une expansion et une exploitation du crédit, des coupures dans les services publics (services sociaux, services de santé, éducation, etc.), augmentation des coûts du logement, etc. Par ailleurs, les grandes entreprises jouent un rôle de plus en plus actif et direct sur la scène politique. On a vu récemment le gouvernement fédéral sentir le besoin de redéfinir sa politique de lobbying afin de garantir une meilleure collaboration avec les "vice-présidents pro-forma", en poste à Ottawa à titre de lobbyistes officiels. (P.-A. COMEAU, Le Devoir, 15 janvier 1986.) Ils peuvent forcer la main aux politiciens pour l'augmentation des budgets militaires (ce qui signifie plus de profits par la fabrication d'armes), pour la privatisation de secteurs-clés contrôlés par l'État (par exemple, Air Canada), pour le développement de "l'actionnariat populaire" (Régime d'épargne-actions, Fonds de solidarité de la Fédération des travailleurs du Québec, etc.), campagne de 
démystification de la Bourse pour une gestion publique calquée sur le modèle des grandes sociétés transnationales, etc. En d'autres termes, le grand capital a maintenant les moyens de pousser le raffinement de l'exploitation à un point tel qu'il est devenu souvent très difficile de cerner la nature des enjeux qui sont au cœur des intérêts entre les couches dominées et la classe dominante.

En poussant le plus loin possible la différenciation des salaires, la diversification apparente des couches sociales, la course aux heures supplémentaires, le conditionnement publicitaire des besoins, l'encouragement à la participation à l'entreprise par des mesures secondaires (formation de clubs sociaux propres à l'entreprise, d'équipes de hockey, boni, fonds de pension, utilisation de techniques de relations humaines, etc.) et par une foule d'autres tactiques, on développe à l'extrême la compétition entre les individus et on transforme la conscience de l'individualité en individualisme, en agir individuel opposé aux projets collectifs. Parallèlement, on encourage la diffusion, sur un même pied d'égalité, de toutes les idéologies prônant l'intégration des travailleurs et des travailleuses à la société.

Actuellement, les fortes pressions des sociétés transnationales sur les gouvernements créent les conditions pour que les luttes ouvrières et populaires s'organisent de plus en plus autour d'enjeux qui confrontent directement le pouvoir étatique, le pouvoir de la classe dominante et du capital monopoliste contre les couches dominées.

En apparence, les luttes populaires et ouvrières peuvent sembler loin du pouvoir ; pourtant, de nouveaux enjeux (paix et désarmement, protection de l'environnement, droits et libertés fondamentales, droits socio-économiques, droits des consommateurs, etc.) confrontent directement l'État et les classes dominées. Hamel et Léonard ont déjà débattu la question en situant l'État comme "enjeu principal du pouvoir et de sa conquête" (1981, p. 55). Par contre, ils constataient que "les mobilisations populaires s'inscrivent souvent à l'intérieur d'un registre d'autogestion ou de démocratie directe qui n'a, somme toute, que peu de choses à voir avec l'idée de conquête du pouvoir d'État." (Id., p. 55.) Certes, la plupart des luttes actuelles ne visent pas directement la prise du pouvoir de l'État, mais elles contribuent, par des affrontements plus directs avec lui, au développement d'une conscience politique à partir d'enjeux chargés d'un contenu à caractère politique. Par exemple, dans un article intitulé à juste titre "Le point de départ : une lutte", Denise Ventelou a donné tout son sens à une lutte locale en y identifiant un enjeu politique.

"Dans son action à propos de la taxe d'eau, I'A.D.D.S. a voulu contester la perte d'un droit acquis et réclamer une augmentation des 
taux de l'aide sociale, mais aussi par delà et à travers ces deux aspects, provoquer l'éveil d'une conscience de classe chez les assistés sociaux: la taxe d'eau, d'objet de lutte devient moyen de pression, puis catalyseur d'une reconnaissance par les assistés sociaux de ce qu'ils vivent et d'une recherche des causes de leur situation dans les structures sociales." (Ampleman et al., 1983, pp. 17-18)

Si, dans les pratiques, beaucoup de luttes portent d'abord sur des questions reliées aux conditions de vie, il faut quand même accorder une place de plus en plus significative à celles qui ont un contenu politique évident par lui-même.

\section{Des luttes anciennes, de nouveaux enjeux}

La promotion et la défense des droits fondamentaux (sociaux, économiques, et politiques) des couches dominées sont devenues un enjeu central de toutes les grandes luttes du mouvement ouvrier et populaire. Parmi ceux qui sont au cour des luttes actuelles: le droit à la vie, à la paix, au travail, à la santé, à l'éducation, à l'autodétermination et à la sécurité et à la solidarité internationale ne sont que quelques-uns de ceux qui sont devenus mobilisateurs, au cours des dernières années. Les droits socio-économiques sont étroitement reliés aux conditions de vie, donc aux politiques sociales. Ainsi, plusieurs catégories des couches dominées (jeunes, assistés(es) sociaux(ales), retraités(es), personnes handicapées, femmes des milieux ouvriers et populaires, travailleurs et travailleuses immigrants) situent ces droits au cœur de leurs luttes. À travers les organisations vouées à la défense de leurs droits, tous ces secteurs participent de plus en plus aux efforts de paix. Les droits socio-politiques portent aussi des enjeux importants: les droits à la protection comme citoyen et citoyenne à la sécurité, à l'information, et ceux d'association et de négociation. Évidemment, cette liste n'est pas exhaustive, ni nouvelle.

\section{Paix et développement : des enjeux-types}

Lorsque l'on parle des droits fondamentaux, il faut faire les liens avec la conjoncture nationale et internationale. Au cours des dernières années, les questions de la faim dans le monde et de la course aux armements ont été très largement diffusées par les médias et sont devenues objets de débat, tant sur la scène des grands forums internationaux que dans chaque pays de la planète et dans chaque ville 
et village. Le droit à la vie et à la paix comme premiers droits de la personne sont devenus des enjeux fondamentaux, traités la plupart du temps de façon inséparable. Cet exemple illustre l'élargissement de l'action militante au cours des années 1980 et un dépassement de la conscience "localiste" vers une conscience et une mobilisation à l'échelle mondiale.

$\mathrm{Si}$, au cours des années 1970 , les militants et militantes pour le droit à la paix et à la vie véhiculaient des slogans souvent à saveur strictement individualiste et écologique (Peace and Love !, Faites l'amour, pas la guerre! etc.), actuellement les slogans semblent les signes d'un niveau de prise de conscience plus aigu des dimensions politiques, économiques et idéologiques de ce droit à la paix et à la vie: Emplois et paix!, Pour le droit à la vie et à la paix!, Le premier droit de la personne!, Pour le droit à la paix et le droit à la vie!, Pas d'armes nucléaires ni à l'Est ni à l'Ouest ! etc.

Au Canada et au Québec, la lutte fut habituellement un enjeu porteur de revendications politiques et humanitaires souvent véhiculées par quelques groupes «spécialisés» dans l'action pour la paix : contre la guerre au Viêt-nam (jusqu'en 1975), pour le désarmement et contre le nucléaire. Présentement, à peu près tous les secteurs de la société (groupes pour la paix, églises, syndicats, groupes populaires, associations professionnelles, etc.) considèrent que cette question constitue un enjeu fondamental. Et pour cause! Les politiques des pays de I'OTAN se basent sur une politique de dissuasion, c'est-à-dire sur une accumulation massive d'armes dans le but de dissuader un ennemi éventuel de nous attaquer. Une telle politique mène tout droit à une augmentation des budgets militaires (400 milliards aux États-Unis et 10 milliards au Canada actuellement, alors qu'au niveau mondial nous atteindrons les 850 milliards en 1986) et à la diminution des budgets à l'aide au développement international et aux politiques sociales. Par exemple, une des premières mesures adoptées par le gouvernement fédéral après l'élection de Brian Mulroney, en septembre 1984, fut l'augmentation de $\$ 6$ millions des budgets militaires et une diminution de l'aide au développement international du même montant! Ce geste illustre où sont les priorités...

L'augmentation des budgets militaires signifie aussi plus de profits pour les marchands de canons, plus de recherches au niveau de la haute technologie, plus de matières premières consacrées au potentiel de destruction et plus de sociétés transnationales impliquées dans le processus de la production d'armes. Aux États-Unis seulement, cent cinquante-deux sociétés sont autorisées à fabriquer et à exporter de ces armes (Bouzouiev, 1984, p. 37). Au Canada, les Accords sur le 
développement de la défense et le partage de la production (D.P.S.A.) visent à intégrer la production militaire canado-américaine à la planification militaire entre le Canada et les États-Unis. Cet accord existe depuis les années 1950 et permet un "libre-échange" des biens militaires entre les deux pays, en particulier dans le cadre des contrats militaires américains avec des industries canadiennes. Évidemment, tout le débat actuel sur le libre-échange est très fortement influencé par cet accord.

Dans une telle conjoncture, le maintien de l'inflation et d'un taux de chômage élevé et la détérioration des conditions de vie ont amené les organisations du mouvement ouvrier et populaire à faire des relations entre l'augmentation des budgets militaires et la diminution de ceux à caractère social. II n'est pas rare maintenant de voir l'utilisation de comparaisons de chiffres pour bien faire saisir les liens entre ces sommes gaspillées en armements et les budgets sociaux.

À cause de leur importance au Canada, la lutte pour les droits à la paix et à la vie sont en train de se donner un ancrage solide dans divers secteurs autour d'enjeux concrets : les essais des missiles de croisière sur le territoire canadien, la conversion des budgets militaires en budgets de développement social, l'autonomie du gouvernement canadien par rapport au gouvernement américain, le renouvellement du contrat de NORAD (North American Defence Command), la participation du Canada au projet "Initiative de défense stratégique" (I.D.S.) - mieux connu comme le projet "guerre des étoiles" - à travers les centres de recherche et les entreprises privées.

Depuis 1983, diverses coalitions se sont formées, et la plus significative a certes été la Campagne de la caravane de la pétition pour la paix (C.C.P.P.), en 1984. Au Québec seulement, environ deux cents organismes différents ont participé au mouvement : les trois centrales syndicales en tête de liste, l'Assemblée des Évêques, la société SaintJean-Baptiste, le Conseil québécois de la paix, et des centaines d'autres. En Colombie-Britannique et en Ontario, la campagne a été encore plus significative en termes de concertation entre les organisations du mouvement ouvrier et populaire et le mouvement pour la paix. À Vancouver, par exemple, regroupé dans une coalition appelée E.A.R. (End the Arms Race), le Conseil de ville et la Commission scolaire ont joué un rôle important à côté des groupes pour la paix, des syndicats, des groupes populaires et d'autres organisations. Un tel exemple illustre de façon concrète comment des enjeux fondamentaux comme le droit à la paix et à la vie sont devenus mobilisateurs et rejoignent de plus en plus l'opinion publique en vue de changements dans les politiques canadiennes (Langille, 1985, pp. 28-29). 
Cette coalition a amorcé une pétition quif devait se faire par le porte-à-porte dans toutes les villes, de même que sur les lieux de travail. La tactique était peut-être un peu téméraire, mais elle a réussi dans une large mesure et a permis un travail à la base. Finalement, la représentativité de la C.C.P.P. a facilité le travail dans l'opinion publique à travers les médias et lors de la clôture de la campagne et le dépôt des pétitions auprès de $M$. Mulroney, car tous les grands médias du pays avaient couvert l'événement.

En résumé, l'action des organisations du mouvement ouvrier et populaire, face à la menace nucléaire et à l'augmentation constante des budgets militaires, a amené l'opinion publique à prendre conscience que les priorités économiques de nos gouvernements vont plus vers la satisfaction des intérêts des grandes sociétés transnationales (le budget fédéral 1985-1986 en est un bel exemple) que vers l'amélioration des conditions de vie et un développement international juste. Quand les centrales syndicales mènent des recherches et des campagnes pour analyser la faisabilité et l'impact de la conversion des usines d'armements en usine de production de biens de consommation civils, cela signifie que la paix et le développement comme enjeux fondamentaux sont entrés au cœur des luttes du mouvement ouvrier et populaire. Les répercussions d'une si vaste campagne restent encore à mesurer, mais d'ores et déjà des actions concrètes traduisent une maturité politique collective de plus en plus grande et une capacité de mobilisation de plus en plus significative. Ainsi, à Toronto, du 8 au 11 novembre 1985, les organisations qui avaient participé à la pétition pour la paix en 1984, jointes à de multiples autres (deux cent trente-trois au total) fondaient l'Alliance canadienne pour la paix autour d'enjeux fondamentaux explicités dans la déclaration d'unité adoptée lors de ce congrès historique.

\section{«I. DÉCLARATION D'UNITÉ}

L'ALLIANCE CANADIENNE POUR LA PAIX (A.C.P.) veut impliquer les Canadiens dans le mouvement mondial pour arrêter la course à l'armement, favoriser le règlement non violent des conflits et garantir la sécurité et le bien-être de tous les peuples. Elle veut faire du Canada un agent de paix dans la communauté des nations qui prenne des initiatives concrètes pour se retirer de la course à l'armement et encourager les initiatives réciproques dans les autres nations.

Le but poursuivi par l'A.C.P. est d'amener le public et le gouvernement canadiens à donner leur appui aux objectifs suivants :

1. L'implication de la population canadienne dans le mouvement mondial pour un monde désarmé, sécure et économiquement juste pour tous. 
2. Un gel et une réduction accélérée négociée et vérifiable de la course aux armements qui mettent fin à la prolifération verticale et horizontale des armements nucléaires et autres sur la terre et dans l'espace.

3. La reconnaissance du Canada comme Zone Libre d'armement (ZLAN) mettant ainsi fin à tout financement, recherche, transport, essai, production et déploiement de systèmes d'armement nucléaire, en tout ou en partie au Canada ainsi que mettant fin à l'exportation de la technologie nucléaire et des combustibles radioactifs qui pourraient être utilisés pour la production des armements nucléaires.

4. L'arrêt du gaspillage à des fins militaires au profit de la satisfaction des besoins humains à travers un programme de conversion et le réentraînement dans le but de promouvoir une économie de paix.

5. La création et la consolidation d'institutions et de mécanismes à l'échelle planétaire pour la prévention des agressions, la solution pacifique des conflits internationaux et la promotion de l'amitié parmi les peuples.

6. La dissolution de tout pacte militaire.

7. L'adoption par le Canada d'une politique de paix autonome.

La présente confrontation internationale menace de précipiter le monde dans une guerre nucléaire. Tous les états en possession d'armes nucléaires, et notamment les deux superpuissances, ont une responsabilité particulière pour mettre fin à l'escalade nucléaire, appliquer le désarmement et fixer les conditions d'une paix mondiale juste. La population de tous les pays a le droit et la responsabilité d'exercer toute son influence sur son propre gouvernement comme sur celui des autres nations pour atteindre ces objectifs."

Le Québec n'est pas en reste puisque l'Alliance pour la paix du Québec existe, basée sur les mêmes principes et les mêmes enjeux.

\section{Solidarité internationale : des enjeux mobilisateurs}

La solidarité entre les organisations ouvrières et populaires du monde entier est une condition essentielle à la sécurité internationale ; à un développement social et économique plus juste; au respect des droits fondamentaux, socio-économiques et socio-politiques; à la création des conditions de la conquête ou du maintien du pouvoir ouvrier et populaire dans plusieurs pays du globe.

Depuis le début des années 1980, les luttes de solidarité internationale se sont consolidées à partir de la menace constante que les forces impérialistes font planer sur les pays en voie de développement. La conquête du pouvoir par les Sandinistes, en 1979, l'avance du F.M.L.N. au Salvador, les progrès du socialisme en Afrique, en Asie et au Moyen-Orient les ont rendues plus agressives et plus menaçantes. Les manifestations en sont parfois évidentes et dramatiques : renversement 
du gouvernement populaire de La Grenade en 1983, aide substantielle aux contras au Nicaragua, formation et maintien de gouvernements militaires de droite dans plusieurs pays d'Amérique latine (Chili, Haïti, Honduras, Guatémala, etc.), en Asie (Philippines, Thaillande, Corée du Sud, etc.), en Afrique (soutien à l'Afrique du Sud, etc.). Toutes ces opérations à l'étranger sont la plupart du temps accompagnées de violations des droits de la personne et de droits fondamentaux comme l'autodétermination. La réaction brutale des forces armées de plusieurs pays contre leurs ouvriers et leurs quartiers traduit le syndrome de la peur devant la montée des forces populaires. On justifie cette agressivité par la peur de perdre le contrôle des pays en voie de développement, donc de perdre les avantages de la domination économique et politique. On le constate par la volonté des pays de contrôler leurs ressources naturelles en opposition à la concentration des richesses entre les mains de sociétés transnationales:

"Cette concentration [celle des sociétés transnationales] ne provient pas seulement d'un intérêt économique particulier des transnationales pour ces pays. Les intérêts militaro-politiques des États-Unis interviennent aussi, de même que l'importance stratégique de certains de ces pays et la volonté de tenir tête au système socialiste de l'économie mondiale. Ce qui entre aussi en ligne de compte, c'est la lutte des jeunes États pour la restauration de la souveraineté nationale sur leurs ressources naturelles et leur activité économique." (Oulianovski, 1982, p. 149.)

Cette lutte pour le droit à l'autodétermination des pays en voie de développement se traduit par des mesures concrètes qui inquiètent les pays développés. Au cours de la période 1970-1976 seulement, les nationalisations de sociétés transnationales ont pris beaucoup d'ampleur, et ce grâce aux luttes des forces progressistes dans plusieurs pays.

" 521 filiales des monopoles britanniques, 342 américaines, 146 françaises et 438 d'autres pays furent nationalisées." (United Nations, 1978, pp. 237 et 254.)

L'acuité et l'âpreté des luttes autour d'enjeux fondamentaux a ainsi permis le développement de la solidarité internationale dans plusieurs pays, dont le Canada. Au Québec, le mouvement ouvrier met de plus en plus l'emphase sur la solidarité internationale, surtout par l'intermédiaire du Centre international de solidarité ouvrière (CISO), organisme financé par la Centrale des enseignants du Québec (C.E.Q.) et par la Confédération des syndicats nationaux (C.S.N.). CISO fait un travail de sensibilisation des syndiqués(es) et collabore concrètement à divers projets d'éducation en collaboration avec des pays en voie de 
développement, organise des brigades de travail au Nicaragua, etc. La Fédération des travailleurs du Québec (F.T.Q.) joue aussi un rôle important : par exemple, un syndicat affilié, les Électriciens unis, a déjà organisé deux brigades d'électriciens au Nicaragua. Par ailleurs, de multiples autres organisations non gouvernementales collaborent à une solidarité internationale : Développement et Paix, l'Association québécoise des organismes de coopération internationale (AQOCI), l'Église unie, des organisations de jeunes comme le Regroupement autonome des jeunes (RAJ) et Carrefour international, Carrefour Tiers-Monde, Credi Lanaudière, Rallye Tiers-Monde et le Service universitaire canadien outre-mer (SUCO).

Toutes ces luttes s'appuient sur un certain nombre de dénominateurs communs, dont le principal est certes le rattrapage des pays en voie de développement autour d'enjeux cruciaux pour les années à venir. En premier lieu, un enjeu politique fondamental dévoile et catalyse un potentiel aussi mobilisateur que les luttes de décolonisation dans les années 1950 et 1960 , soit le droit à l'autodétermination des pays en voie de développement, c'est-à-dire le droit à l'indépendance totale aux plans économique, politique et militaire. Deuxièmement, les enjeux économiques sont vitaux : le développement de l'agriculture, de l'industrie et de relations commerciales équitables, l'intervention des sociétés transnationales et le règlement des dettes avec les pays développés. En effet, actuellement, la dette de l'Amérique latine et des Caraïbes atteint les 360 milliards de dollars aux banques privées, aux gouvernements de pays capitalistes avancés, à la Banque mondiale et au Fond monétaire international; et la dette extérieure totale de tous les pays en voie de développement est rendue à 900 milliards (Diaz, 1985, pp. 12-13).

Les luttes autour de tels enjeux commandent la solidarité internationale et prennent une signification concrète depuis quelques années: campagne de vente du crayon et du cahier pour financer le processus d'alphabétisation au Nicaragua, organisée par la C.E.Q. en 1981-1982, organisation de brigades pour le Nicaragua, campagnes populaires pour aider le peuple en lutte au Salvador, manifestation contre la répression au Chili, vigiles hebdomadaires face au consulat d'Afrique du Sud à Montréal, coopération internationale par des subventions et l'envoi de coopérants et des coopérantes et combien d'autres. 


\section{Les droits socio-économiques : des enjeux vitaux et actuels}

Les droits socio-économiques signifient droit à des conditions de vie décentes pour les couches sociales les plus démunies. Au cours des dernières années, plusieurs luttes significatives ont démontré que les organisations s'en tiennent de moins en moins à des revendications locales, mais définissent leurs stratégies autour d'enjeux fondamentaux plus universels comme l'amélioration des conditions de vie, la création d'emplois, le droit à la santé, etc., et autour de liens avec des enjeux qui concernent tous les secteurs de la société.

Ainsi, au cours des dernières années, les luttes menées par le Front commun des assistés sociaux et des assistées sociales et par des Associations pour la défense des droits sociaux (A.D.D.S.) ont mis de l'avant plusieurs droits fondamentaux, même dans des luttes très ponctuelles. Pensons à un slogan clé comme "l'aide sociale, c'est un droit" et à d'autres droits (à sa dignité, au logement, à la santé, au travail) devenus des enjeux clairs dans leurs luttes (Ampleman et al., 1983, p. 64).

Au printemps de 1983, la Grande marche pour l'emploi, organisée par un regroupement des centrales syndicales et plusieurs autres organisations a lancé publiquement un cri d'alarme sur la gravité des conditions du chômage et de l'aide sociale, mais a démasqué en même temps les priorités des gouvernements, tant à Ottawa qu'à Québec. On a réussi à faire ressortir que les intérêts de la classe dominante ne pouvaient conduire à de véritables programmes de création d'emploi ; il est alors vite devenu évident que les divers programmes gouvernementaux favorisaient d'abord le patronat. Par exemple, au plan économique: l'aide aux entreprises, les programmes de création d'emplois (Canada au Travail, etc.); au plan politique : le rôle de l'État dans la recherche de solutions à la crise économique, et par rapport aux entreprises, etc.; au plan idéologique: le plein emploi, les conceptions du développement social et économique du Canada, etc.

Les revendications véhiculées lors de la Grande marche pour l'emploi ne sont pas restées lettre morte chez les jeunes. Dès 1983, un groupe de jeunes impliqué dans l'organisation de la Grande marche mettait sur pied le Regroupement autonome des jeunes (RAJ). Organisme de lutte pour l'amélioration de leurs conditions de vie, le RAJ décide vite de passer à l'action politique directe en présentant un candidat dans le comté de Saint-Jacques. Il lie ses revendications à celles des autres catégories de gens auxquels les gouvernements n'offrent pas d'alternatives politiques. Son discours est assez explicite : 
"Nous, les jeunes, actuellement regroupés au sein du RAJ, nous sommes déterminés-es à remporter la bataille que nous livrons au gouvernement pour la satisfaction de nos revendications. À ce sujet, nous sommes convaincus d'avoir l'appui de la majorité de la population, puisque les problèmes que nous vivons, ce sont ceux des travailleurs-ses, des chômeurs-ses, des femmes qui sont quotidiennement opprimées, de tous ceux et celles qui voudraient travailler convenablement, mais qui se retrouvent confiné-es à la pauvreté parce que les patrons des compagnies, les politiciens et les gouvernements en ont décidé ainsi. " (RAJ, 1984, pp. 1-2.)

Dans son action, le RAJ met l'emphase sur les droits socioéconomiques, autour d'enjeux clés comme la parité dans l'emploi et la création d'emploi, mais aussi sur les droits socio-politiques:

"Si les gouvernements prétendent relancer l'économie canadienne et québécoise suite aux années de crise, ils se préservent bien cependant de relancer également les droits démocratiques de la population. C'est tout le contraire qui se produit. Nous assistons à la valorisation de certaines conduites autoritaires et répressives qui appellent à une dénonciation de tous les instants de la part des jeunes et de l'ensemble des organisations progressistes. » (RAJ, 1984 p. 15.)

Dans leur analyse, et c'est un phénomène relativement nouveau dans les luttes des jeunes au Québec, ceux du RAJ englobent aussi les questions internationales en faisant des liens étroits avec leur propre sort ici, au Québec, au cœur d'un pays dit développé. La paix et la solidarité internationale ont une place aussi importante parmi les enjeux que leur lutte pour de meilleures conditions de vie.

"Plus le Canada mise sur la course aux armements pour assainir son économie, plus les masses canadiennes en souffrent, et plus le gouvernement se trouve lié à la logique militariste américaine qui fait qu'il adopte lui aussi des positions interventionnistes contre des peuples qui s'opposent aux visées sanglantes de Reagan en Amérique centrale et ailleurs." (RAJ, p. 21.)

Au niveau des luttes pour les droits socio-économiques, si les jeunes mènent des actions très politisées, les retraités(es) ne sont pas en reste. Depuis cinq ans, l'Association québécoise pour la défense des droits des retraités(es) (A.Q.D.R.) mène la lutte autour d'un enjeu clé : l'amélioration de leurs conditions de vie. Elle utilise des moyens de pression (par exemple la fameuse manifestation sur la colline parlementaire à Ottawa en 1983, manifestation haute en couleur parce que basée sur l'exposition d'immenses bannières réalisées par elles, des moyens d'éducation populaire (sessions de formation, ateliers de créativité "conscientisante", comme la préparation de l'exposition de 
bannières), l'analyse de leur situation (manifeste, mémoires, etc.) et l'utilisation de l'opinion publique, etc.

Les membres de I'A.Q.D.R. sont conscients que le dépassement des activités de loisirs pour les ex-ouvriers et ouvrières est une nécessité pour réaliser des gains. Ils voient aussi l'importance de faire des liens avec des enjeux plus globaux, comme la paix et le développement, le respect des droits démocratiques et le contrôle du pouvoir politique.

\section{Les droits socio-politiques : des enjeux réels}

De prime abord, les droits socio-politiques ne sont pas aussi mobilisateurs que ceux d'ordre socio-économiques parce qu'on a souvent l'impression qu'ils ne concernent pas les individus dans leur vie de chaque jour. Pourtant, la réalité est bien différente. Par exemple, la lutte des centrales syndicales pour le droit de négocier dans le secteur public est une lutte actuelle autour d'un enjeu, soit celui d'exercer son droit d'association. Celui-ci est remis en question par rapport aux employés(es) de l'État qui sont syndiqués(es) mais en réalité, ce n'est que le fer de lance de l'offensive patronale et étatique contre le mouvement ouvrier, attaquant le droit d'association par toutes sortes de mesures plus ou moins subtiles (réforme du Code du travail; poursuites contre les travailleurs et travailleuses en grève, comme à Marine Industries, etc.).

Si les droits des travailleuses et des travailleurs syndiqués sont menacés, les droits démocratiques des citoyens et citoyennes du Canada le sont tout autant. On se rappelle l'illustre Commission Keable, chargée d'enquêter sur les agissements de la Gendarmerie royale du Canada au Québec, qui a réussi à lever une partie du voile sur l'illégalité de l'agir policier en maintes circonstances ( $v o l$ de listes des membres du P.Q., infiltration du F.L.Q., etc.). Mais, depuis, les méthodes se sont raffinées et on a réussi à légaliser des stratégies policières autrefois défendues par la Loi C-9 adoptée le 21 juin 1984; et, avec les moyens électroniques dont disposent maintenant les corps policiers, il est devenu très facile de contrôler et suivre à la trace tous les militants et toutes les militantes du mouvement ouvrier et populaire et de la gauche politique en général, en somme de toutes les personnes "susceptibles de porter atteinte à la sécurité de l'État" se plaisent à répéter les politiciens et politiciennes.

La création de l'Agence de renseignement du gouvernement fédéral a comme objet de bien suivre les 800000 dossiers politiques d'individus à travers le Canada, et ce de façon légale et efficace en 
ouvrant le courrier, en pratiquant l'écoute électronique, en effectuant des entrées par effraction s'il le faut, en consultant des dossiers confidentiels (médicaux, d'avocats, de notaires, de crédit, d'employés(es), etc.)

L'enjeu porte sur la liberté d'opinion, la liberté d'option et d'action politiques. Plus les luttes pour les droits socio-économiques et fondamentaux "se politisent ", plus l'État réagit par un contrôle répressif accru. Cette question interpelle donc le mouvement ouvrier et populaire et amène les militants et les militantes à faire des liens entre les divers enjeux. Penser lutter pour ces droits est devenu un crime qui n'épargne personne, surtout pas les couches dominées. Au total, c'est l'apparition du délit d'opinion en droit canadien. Une opinion est plus dangereuse qu'un crime aux yeux de notre gouvernement.

\section{Conclusion}

À travers une trop brève analyse, on constate, de plus en plus, et c'est peut-être là un trait caractéristique de l'action militante des années 1980, que des enjeux fondamentaux confrontent les militants et les militantes. Nous sortons des luttes sectorielles et ponctuelles, menées par des organisations limitées aux seules revendications concernant leur groupe, pour lier davantage les diverses luttes autour d'enjeux qui les recoupent et impliquent une analyse et une action politique de plus en plus directe.

Dans tous les secteurs, des dizaines d'intervenants et d'intervenantes en organisation communautaire sont au cœur de l'action militante, que ce soit à I'A.Q.D.R., au RAJ, à la Ligue des droits et libertés, dans le mouvement pour la paix, les A.D.D.S., les comités de solidarité, etc. Contrairement à l'opinion courante, qui prétend que l'action militante est morte, les pratiques nous démontrent le contraire et nous révèlent la perception d'enjeux porteurs de dimensions locales certes, mais aussi nationales et internationales.

\section{Références bibliographiques}

ALINSKI, S., Manuel de l'animateur social, Paris, Seuil, 1976, 250p.

Alliance canadienne pour la paix, Document sur la structure, rapport du Congrès tenu à Toronto, 8-11 mai 1985, 2p. 
Ampleman, G. et al., Pratiques de conscientisation. Expériences d'éducation populaire au Québec, Montréal, Nouvelle Optique, 1983.

Ampleman, G., "Le bien-être social: pas un choix, mais un droit", dans: G. AMPLEMAN et al., op. cit. : 41-76.

Bouzoulev, A., Les sociétés trans-nationales et le militarisme, Moscou, Éditions du progrès, 1984, 365p.

Claude, H., La $3^{\circ}$ course aux armements, Paris, Éditions sociales, 1982, 203p.

Comeau, P.-A., "Lobbying et morale», Le Devoir, 15 janvier 1986, p. 8.

Cunningham, M. et M. Fitzpatrick, How the New Technology Is Leading Us to War, New York, Warner Brooks, 1983, 274p.

DiAz, C., "La bomba del tiempo de la deuda externa latino-americana", Araucaria, no 31, 1985 : 11-13.

DORÉ, G., Le jeu des forces sociales dans nos luttes : analyse de la conjoncture, Québec, GRAP, cahier no 4, 1981, pp. 21-23.

Emelianov, T., Un business dangereux, Moscou, Agence Novosti, 1983, 75p.

HAMEL, P. et J.-F. LÉONARD, Les organisations populaires, l'État et la démocratie, Montréal, Nouvelle Optique, 1981, 208p.

HARNECKER, M., Les concepts élémentaires du matérialisme historique, Bruxelles, Contradictions, 1974, 258p.

LAmoureux, H., R. MAyer et J.-P. PANet-RAymOnd, L'intervention communautaire, Montréal, Éditions Saint-Martin, 1984, 237p.

LANGILlE, D., "Strategies for the Canadian peace movement ", Canadian Dimension, vol. 19, no 1, 1985 : 27-31.

OUlianovski, R., "Le néo-colonialisme : formes et méthodes". Sciences sociales, no 4, 1982: 147-160.

Regroupement autonome des jeunes, Le RAJ dans Saint-Jacques : pour la parité des emplois, Montréal, RAJ, 1984, 27p.

United Nations, Transnational Corporations in World Development: A Reexamination, New York, O.N.U., Economic and Social Council, 1978, pp. 237-254.

Ventelou, D., "Le point de départ : une lutte", dans : G. Ampleman et al., op. cit. : 15-40. 